\title{
The suppression of Brd4 inhibits peripheral plasma cell differentiation and exhibits therapeutic potential for systemic lupus erythematosus
}

\author{
Shan Zeng ${ }^{1}$, Qian Qiu ${ }^{1}$, Yi Zhou ${ }^{1}$, Youjun Xiao ${ }^{1}$, Jingnan Wang ${ }^{1}$, Ruiru Li $^{1}$, Siqi Xu${ }^{1}$, \\ Maohua Shi ${ }^{1}$, Cuicui Wang ${ }^{1}$, Yu Kuang ${ }^{1}$, Minxi Lao ${ }^{1}$, Xiaoyan Cai ${ }^{1}$, liuqin liang ${ }^{1}$, and \\ Hanshi $\mathrm{Xu}^{1}$ \\ ${ }^{1}$ Sun Yat-Sen University
}

April 14, 2021

\begin{abstract}
Background and purpose: To investigate the role of bromodomain-containing protein 4 (Brd4) in regulating B cell differentiation and its therapeutic potential for B cell-mediated autoimmune diseases such as systemic lupus erythematosus (SLE). Experimental Approach: Human and murine B cells were purified and cultured with different stimuli. B cell surface markers, proliferation and apoptosis were estimated by flow cytometry. Gene expression was measured by quantitative real-time PCR. Brd4 binding sites were analysed by the luciferase reporter assay and the chromatin immunoprecipitation (ChIP) assay. PFI-1 or JQ1 was used to inhibit Brd4. Mice with B cell-specific deletion of the Brd4 gene (Brd4flox/floxCD19-Cre+/-) and MRL/lpr mice were used to perform the in vivo experiments. Key Results: Brd4 inhibition suppressed plasmablast-mediated plasma cell differentiation but did not influence proliferation or apoptosis in healthy human and murine CD19+ B cells. PFI-1 treatment reduced the secretion of IgG and IgM in the supernatants of costimulation-induced B cells. Mechanistically, Brd4 regulates the terminal differentiation of B cells into plasma cells by targeting BLIMP1 by directly binding and activating the endogenous BLIMP1 promoter. Interestingly, PFI-1 treatment decreased the percentages of plasmablasts and plasma cells from patients with SLE. PFI-1 administration reduced the percentages of plasma cells, hypergammaglobulinemia and attenuated nephritis in MRL/lpr mice. Pristane-injected Brd4flox/floxCD19-Cre+/- mice exhibited improved nephritis and reduced percentages of plasma cells. Conclusions and Implications: Brd4 is an essential factor in regulating plasma cell differentiation. Brd4 inhibition may be a potential new strategy for the treatment of B cell-associated autoimmune disorders, including SLE.
\end{abstract}

The suppression of Brd4 inhibits peripheral plasma cell differentiation and exhibits therapeutic potential for systemic lupus erythematosus

Shan Zeng ${ }^{1,2, a}$, Qian Qiu ${ }^{1, a}$, Yi Zhou ${ }^{1, a}$, Youjun Xiao ${ }^{1}$, Jingnan Wang ${ }^{1}$, Ruiru $\mathrm{Li}^{1}$, Siqi Xu ${ }^{1}$, Maohua Shi ${ }^{1}$, Cuicui Wang ${ }^{3}$, Yu Kuang ${ }^{1}$, Minxi Lao ${ }^{1}$, Xiaoyan $\mathrm{Cai}^{3{ }^{3}}{ }^{*}$, Liuqin Liang ${ }^{1,}{ }^{*}$, Hanshi $\mathrm{Xu}^{1,{ }^{*}}$

${ }_{1}^{1}$ Department of Rheumatology and Immunology, The First Affiliated Hospital, Sun Yat-sen University, Guangzhou, Guangdong, China

2 Department of Rheumatology, The First Affiliated Hospital, Jinan University, Guangzhou, Guangdong, China

${ }^{3}$ Department of Rheumatology, Guangzhou First People's Hospital, School of Medicine, South China University of Technology, Guangzhou, Guangdong, China

${ }^{\text {a }} \mathrm{ZS}, \mathrm{QQ}$ and ZY contributed equally to this work

Running Title: Brd4 and plasma cell in SLE 
Key Words: SLE, BRD4, B cell, PFI-1

word count: 5334

* Correspondence and requests for materials should be addressed to:

Hanshi Xu, M.D., Ph.D. E-mail: xuhanshi@mail.sysu.edu.cn

Liuqin Liang, M.D, E-mail: lliuq@mail.sysu.edu.cn

Xiaoyan Cai, M.D, E-mail: caixytracy@126.com

\section{ABSTRACT}

Background and purpose: To investigate the role of bromodomain-containing protein 4 (Brd4) in regulating B cell differentiation and its therapeutic potential for B cell-mediated autoimmune diseases such as systemic lupus erythematosus (SLE).

Experimental Approach: Human and murine B cells were purified and cultured with different stimuli. B cell surface markers, proliferation and apoptosis were estimated by flow cytometry. Gene expression was measured by quantitative real-time PCR. Brd4 binding sites were analysed by the luciferase reporter assay and the chromatin immunoprecipitation (ChIP) assay. PFI-1 or JQ1 was used to inhibit Brd4. Mice with B cell-specific deletion of the $\operatorname{Brd} 4$ gene $\left(\mathrm{Brd} 4^{\text {flox} / f l o x} \mathrm{CD} 19-\mathrm{Cre}^{+/-}\right)$and $\mathrm{MRL} / \mathrm{lpr}$ mice were used to perform the in vivo experiments.

Key Results: Brd4 inhibition suppressed plasmablast-mediated plasma cell differentiation but did not influence proliferation or apoptosis in healthy human and murine CD19+ B cells. PFI-1 treatment reduced the secretion of $\operatorname{IgG}$ and IgM in the supernatants of costimulation-induced B cells. Mechanistically, Brd4 regulates the terminal differentiation of $\mathrm{B}$ cells into plasma cells by targeting BLIMP1 by directly binding and activating the endogenous BLIMP1 promoter. Interestingly, PFI-1 treatment decreased the percentages of plasmablasts and plasma cells from patients with SLE. PFI-1 administration reduced the percentages of plasma cells, hypergammaglobulinemia and attenuated nephritis in MRL/lpr mice. Pristane-injected $\mathrm{Brd} 4{ }^{\text {flox}} /{ }^{\text {flox }} \mathrm{CD} 19-\mathrm{Cre}^{+/-}$mice exhibited improved nephritis and reduced percentages of plasma cells.

Conclusions and Implications: Brd4 is an essential factor in regulating plasma cell differentiation. Brd4 inhibition may be a potential new strategy for the treatment of B cell-associated autoimmune disorders, including SLE.

Abbreviations: Brd4, bromodomain-containing protein 4; SLE, systemic lupus erythematosus; BLIMP1, B lymphocyte-induced maturation protein 1; XBP1, X-box-binding protein 1; IRF4, interferon regulatory factor 4

\section{What is already known}

Systemic lupus erythematosus (SLE) is an autoimmune disorder characterized by multiple abnormality of B cell.

BET bromodomain inhibition modulates adaptive immune responses.

\section{What this study adds}

BRD4 regulate the differentiation of B cell subsets by regulating BLIMP1.

\section{What is the clinical significance}

BRD4 is an essential factor in regulating plasma cell differentiation.

BRD4 protein inhibitor PFI-1 have therapeutic potential in B cell-associated autoimmune disorders including SLE.

\section{INTRODUCTION}


The generation of antibodies is one of the most important roles of B cells and is the basis for autoantibodymediated autoimmune disorders. The modulation of antibody production is associated with the generation and maintenance of terminally differentiated B cells, known as plasmablasts and plasma cells, from their B cell precursors(Carsetti, Rosado et al., 2004; Nutt, Hodgkin et al., 2015). Activated B cells have the ability to proliferate, undergo immunoglobulin class-switch recombination, and differentiate into plasmablasts that express high levels of IRF4 and X-box-binding protein 1 (XBP1) and medium levels of B lymphocyte-induced maturation protein 1 (BLIMP1)(Radbruch, Muehlinghaus et al., 2006; Shlomchik \& Weisel, 2012). BLIMP1 is a critical transcriptional repressor that promotes the terminal differentiation of B cells and plays an essential role in the formation of mature plasma cells (Nutt, Taubenheim et al., 2011; Shapiro-Shelef, Lin et al., 2003). However, the mechanisms that control B cell terminal differentiation are complex and remain incompletely understood.

Systemic lupus erythematosus (SLE) is an autoimmune disorder characterized by increased levels of pathogenic autoantibodies. Multiple defective checkpoints during B cell development, maturation, and activation have been demonstrated in SLE patients (Cappione, Anolik et al., 2005; Yurasov, Tiller et al., 2006; Yurasov, Wardemann et al., 2005) and mouse lupus models(Grimaldi, Cleary et al., 2002; Grimaldi, Michael et al., 2001; Santulli-Marotto, Qian et al., 2001). It is well recognized that B cells are important for both the initiation and maintenance of autoimmunity in SLE, and B cell-targeting therapies may be beneficial for patients with SLE(Parodis, Stockfelt et al., 2020; Reddy, Cambridge et al., 2015).

As a family of epigenetic adaptors, bromodomain and extraterminal domain (BET) proteins (Brd2, Brd3, Brd4, and Brdt) can bind to acetylated lysine residues and link acetylated chromatin and gene transcription (Filippakopoulos, Picaud et al., 2012). BET bromodomain inhibition modulates the differentiation and activation of T cells(Bandukwala, Gagnon et al., 2012; Mele, Salmeron et al., 2013) and dendritic cells (Toniolo, Liu et al., 2015), which suggests that BET proteins may be involved in adaptive immune responses. A previous finding indicated a role of Brd2 in promoting the expansion and mitogenesis of murine B cells (Belkina, Blanton et al., 2014); however, to date, it remains undefined whether BET bromodomains regulate the differentiation of B cell subsets and have therapeutic potential in B cell-associated autoimmune disorders. Because Brd4 is the most studied BET protein, we herein aimed to determine the involvement of Brd4 in regulating the differentiation of B cell subsets and to further explore its possible therapeutic potential for B cell-mediated autoimmune diseases such as SLE.

\section{MATERIALS AND METHODS}

\section{Human blood samples and animals}

All subjects (healthy donors: $\mathrm{n}=18$, active SLE patients: $\mathrm{n}=18$ ) recruited for this study were selected via a protocol approved by the Ethics Committee of the First Affiliated Hospital, Sun Yat-sen University and provided informed consent. All patients with SLE were diagnosed based on the American College of Rheumatology (ACR) classification criteria for SLE (Hochberg, 1997). The characteristics of SLE patients are shown in supplemental Table S1.

All mice were housed in specific pathogen-free conditions, used in accordance with the guidelines and approved by the Institutional Animal Care and Use Committee of the First Affiliated Hospital, Sun Yat-sen University.

\section{Human B cell isolation and cultures}

Venous peripheral blood was withdrawn from each patient and healthy control subject. B cells were isolated by positive selection using CD19 beads, according to the manufacturer's instructions (Miltenyi, Bergisch Gladbach, Germany; routine purity > 95\%). Cell culture was performed in human B cell culture medium (Lonza, Walkersville, MD, USA) supplemented with 10\% foetal bovine serum (FBS) and 1\% penicillin/streptomycin.

\section{B cells stimulation}


B cells were cultured in 96-well plates at $1 \times 10^{6}$ cells $/ \mathrm{ml}$ in $100 \mu \mathrm{l}$ of culture medium alone or supplemented with various cytokines and stimuli: $1 \mu \mathrm{g} / \mathrm{ml}$ anti-CD40 (R\&D Systems), anti-IgM (Jackson ImmunoResearch Laboratories), $50 \mathrm{ng} / \mathrm{ml} \mathrm{IL-4} \mathrm{(R \& D} \mathrm{Systems),} \mathrm{and} \mathrm{various} \mathrm{concentrations} \mathrm{of} \mathrm{PFI-1} \mathrm{(R \& D} \mathrm{Systems).}$

For human plasmablast differentiation, B cells were stimulated with anti-IgM $(5 \mu \mathrm{g} / \mathrm{ml}), \mathrm{rCD} 40 \mathrm{~L}(1 \mu \mathrm{g} / \mathrm{ml})$,

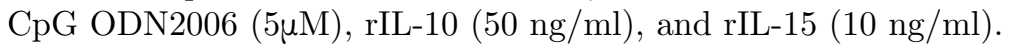

\section{Cell proliferation measurement}

B cells were cultured for 3,4 , or 6 days as mentioned above. Cell proliferation was determined by CFSE (Invitrogen Life Technologies) dilution using fluorescence-activated cell sorting (FACS) analysis. CFSElabelled cells were cultured for 3 to 6 days, and data were collected by FACS analysis.

\section{Cell apoptosis and viability assay}

To determine apoptosis, cultured B cells were double-stained with an annexin V-FITC conjugate and propidium iodide (PI) using TACS annexin V kits (Oncogene) according to the manufacturer's instructions. The stained B cells were analysed using a FACS Calibur flow cytometer (BD Biosciences) within 30 min.

\section{Quantitative real-time PCR}

Total RNA from B cells was isolated by the Takara PrimeScript RT reagent kit according to the manufacturer's protocol. Quantitative real-time PCR (qRT-PCR) was performed using the Bio-Rad CFX96 system. Each sample was processed and analysed in duplicate. The primers used for qRT-PCR are listed in supplemental Table S2. To quantify the relative expression of each gene, Ct values were normalized to the endogenous reference values $(\Delta \mathrm{Ct}=\mathrm{Ct}$ target-Ct GAPDH) and compared with a calibrator using the $\Delta \Delta \mathrm{Ct}$ method $(\Delta \Delta \mathrm{Ct}=\Delta \mathrm{Ct}$ sample- $\Delta \mathrm{Ct}$ calibrator $)$.

\section{Measurement of B cell surface markers}

$\mathrm{B}$ cells were harvested and stained with antibodies for cell surface markers and incubated for 20 minutes at $4^{\circ} \mathrm{C}$ in the dark. The samples were examined on a Beckman Coulter flow cytometer, and the data were analysed with FlowJo software (Tree Star, Ashland, Ore).

\section{Establishment of B cell-specific Brd4-deficient mice}

Floxed Brd4 mice (Brd4 $4^{\text {flox/WT }}$ ) were generated by CRISPR/Cas-mediated genome engineering technology (H. Yang, Wang et al., 2014), which was supported by the Nanjing Biomedical Research Institute of Nanjing University (Nanjing, China). The Brd4 gene was subjected to editing in embryos to generate mice carrying conditional alleles. LoxP sites for Cre-mediated recombination were inserted 5' and 3' into the fifth coding exon. B cell-specific Brd4-deficient mice were generated through appropriate breeding of Brd4 4 flox/WT mice with CD19-Cre mice (Stock No: 006785, The Jackson Laboratory) and maintained on a C57BL/6 background. Brd4 ${ }^{\text {flox } / \text { flox }} \mathrm{CD} 19-\mathrm{Cre}^{-/-}(\mathrm{control})$ and $\mathrm{Brd} 4^{\text {flox }} /$ flox $\mathrm{CD} 19-\mathrm{Cre}^{+/-}$(knockout) mice were born at the expected Mendelian ratio, and these mice did not show any gross defects in survival.

\section{Treatment of MRL/lpr mice with the Brd4 inhibitor PFI-1}

Female MRL/lpr mice were purchased from Shanghai SLAC Laboratory Animal Center (Chinese Academy of Sciences). 12 week-old female MRL/lpr mice were randomly divided into 2 groups (8 mice per group). The two groups of mice were intraperitoneally injected daily with PFI-1 $(10 \mathrm{mg} / \mathrm{kg} / \mathrm{d})$ or vehicle.

Mouse urine was collected every week for $24 \mathrm{~h}$ in metabolic cages starting from week 12. Urinary protein, blood urea nitrogen $(\mathrm{BUN})$ and creatinine $(\mathrm{Cr})$ concentrations were determined by assay kits (Jiancheng Biotechnology, Nanjing, China) according to the manufacturer's instructions.

The serum levels of anti-dsDNA antibodies, ANA, complement C3, complement C4, IgG, IgG1, IgG2a and IgM were assayed using ELISA kits (USCN LIFE Science Inc, Wuhan, China) according to the manufacturer's instructions. The percentages of plasma cells $\left(\mathrm{CD} 19^{+} \mathrm{CD} 138^{+}\right)$, naive B cells $\left(\mathrm{CD} 19^{+} \mathrm{IgM}^{+} \mathrm{IgD}^{+} \mathrm{CD} 27^{-}\right)$and memory B cells $\left(\mathrm{CD} 19^{+} \mathrm{IgM}^{+} \operatorname{IgD} \mathrm{CD}^{-} 7^{+}\right)$were detected by flow cytometry. 
Kidney tissues were collected and immersed in $10 \%$ neutral buffered formalin immediately. Paraffin sections were stained with haematoxylin and eosin (HE). Histopathology of glomerular and renal vascular lesions was graded on a scale of 0-3 for severity in a blinded manner.

For the detection of immune complex deposition, kidneys were embedded in OCT compound and snap-frozen at -80degC. Frozen kidney sections $(5 \mu \mathrm{m})$ were fixed in acetone, blocked with $10 \%$ goat serum for 30 minutes, and stained with fluorescein isothiocyanate (FITC)-conjugated goat anti-mouse IgG or mouse complement C3 (Cedarlane, Burlington, Ontario, Canada).

\section{Isolation of mouse splenocytes, lymphocytes and peripheral blood mononuclear cells}

Mice were sacrificed under anaesthesia. The spleens and lymphonodus were removed and dissociated in RPMI-1640 medium (Invitrogen, Carlsbad, CA). The cell suspension was passed through a $70 \mu \mathrm{m}$ strainer, and cells were collected by centrifugation at $300 \mathrm{~g}$ for 5 minutes. PBMCs were harvested by Ficoll density gradient centrifugation.

\section{Pristane-induced murine lupus nephritis model}

A single intraperitoneal injection of $0.5 \mathrm{~mL}$ pristane or PBS using a $1 \mathrm{~mL}$ syringe was applied to 6- to 8-weekold female Brd $4^{\text {flox } / \text { flox }} \mathrm{CD} 19-\mathrm{Cre}{ }^{+/-}$mice or $\mathrm{Brd} 4^{\text {flox } / \text { flox }} \mathrm{CD} 19-\mathrm{Cre}{ }^{-/-}$mice. The mice were housed under specific pathogen-free conditions under a 12-hour light/dark cycle. Serum and urine samples were collected monthly. Spleen, kidney, lymph node, and PBMCs were obtained at 6 months after pristane injection. Kidney histopathologic changes were determined by H\&E staining. No mice died during the course of the experiments.

\section{Luciferase reporter assay}

BLIMP-1 promoter regions were assessed in $3 \mathrm{~kb}$ upstream from the transcription start site of the mouse Blimp-1 sequences. To construct the Blimp-1 Luc luciferase reporter plasmid, approximately $0.9-1 \mathrm{~kb}$ of each sequence of the Blimp-1 promoter region were amplified by PCR using specific primers and subsequently cloned into the pGL4.10 vector (Promega). HEK293T cells were cotransfected with $500 \mathrm{ng}$ of luciferase reporter, and $100 \mathrm{nM}$ of Brd4-siRNA or control-siRNA. After 72 hours of transfection, luciferase activities were measured by the Dual-Luciferase Reporter Assay System (Promega) according to the manufacturer's instructions. The sequences of primer used were listed in supplemental Table S3.

\section{Chromatin immunoprecipitation (ChIP) assay}

ChIP assays were performed using the ChIP Assay Kit (ChIP-IT High Sensitive Kit, Active Motif, CA, USA) according to the manufacturer's instructions. Briefly, cells were cross-linked and lysed and the chromatin was then sheared by sonication to produce fragments from 100 to 500 bps. The complexes were immunoprecipitated using anti-IgG control or anti-Brd4 antibody, followed by incubation with protein G-agarose beads. Cross-links were reversed and immunoprecipitated DNA was purified. The presence of potential Brd4 binding sites was measured by qPCR using the primers listed in supplemental Table S4.

\section{Statistics}

The data are expressed as the means \pm SEM. We performed the experimental procedures and treatment and data analyses under blinded conditions. To reduce the baseline variability between independent experiments, we normalized the quantitative analyses of immunoblots and mRNA expression. The data were normalized as the fold change over the mean of the control. We compared 2 groups by Student's $t$ test; 3 or more different groups were evaluated by one-way analysis of variance (ANOVA), And if F reached significance two post hoc tests were used: the Dunnett post hoc test when comparing each group with control, or the Sidak post hoc test whenever a multiple group comparison was necessary. For in vivo studies, all groups were initially designed to contain 8 or 6 mice. We used nonparametric methods (Kruskal-Wallis test followed by Dunn's post hoc tests when $\mathrm{F}$ reached significance). $P$; 0.05 was considered significant. We performed statistical analyses of the data using SPSS v18.0 software. 


\section{RESULTS}

Effect of Brd4 inhibition on the proliferation and apoptosis of human peripheral activated CD19+ B cells

Initially, we evaluated the effect of Brd4 inhibition on B cell survival. We observed that stimulation with combinations of anti-IgM, rCD40L, CpG ODN2006, rIL-4 and rIL-2 increased the survival of cultured B cells at days 3,5 and 7 (Fig. 1A); however, the Brd4-specific inhibitor PFI-1 did not affect the survival of costimulated B cells. We next determined the effect of Brd4 inhibition on B cell proliferation. We demonstrated that B cells stimulated with anti-IgM/anti-CD40 exerted increased proliferative capacity compared with unstimulated B cells; however, this increase was not inhibited by treatment with PFI-1 (Fig. 1B and C). We also examined the inhibitory effect of PFI-1 on B cell apoptosis, as measured by flow cytometry with annexin V/PI staining. Stimulation with anti-IgM/anti-CD40 decreased the frequency of apoptotic cells, but in cultures of activated B cells, PFI-1 did not increase the percentage of early apoptotic cells at days 3, 5 and 7 (Fig. 1D and E). We also found that treatment with JQ1, another Brd4 inhibitor, did not affect the proliferation and apoptosis of activated human peripheral CD19+ B cells (data not shown). These data suggest that Brd4 is not involved in the proliferation and apoptosis of activated human peripheral CD19+ B cells.

Effect of Brd4 inhibition on the percentages of naïve, memory, plasmablasts, and plasma cells in healthy human CD19+B cells

To determine whether Brd4 inhibition regulates the differentiation of human B cells, purified CD19+ B cells isolated from healthy donors were activated with combinations of anti-IgM, rCD40L, CpG ODN2006, rIL-4 and rIL-2 in the presence or absence of PFI-1, and then the cells were stained for various indicated surface markers. Flow cytometric analysis was performed to determine the percentages of naïve B cells $\left(\mathrm{CD} 19^{+} \mathrm{IgD}^{+} \mathrm{CD} 27^{-}\right)$, memory B cells $\left(\mathrm{CD} 19^{+} \mathrm{IgD}^{-} \mathrm{CD} 27^{+}\right)$, plasmablasts $\left(\mathrm{CD} 19^{+} \mathrm{IgDD}^{-} \mathrm{CD} 27^{++}\right)$and plasma cells $\left(\mathrm{CD} 19^{+} \mathrm{CD} 38^{++} \mathrm{CD} 138^{+}\right)$. As shown in Fig.2A and B, after 5 days in culture, treatment with PFI-1 significantly decreased the percentages of plasmablasts and plasma cells, but did not affect the percentages of naïve and memory B cells. We also observed a similar effect of PFI-1 on B cell differentiation over 7 days in culture (Fig.S2). We also demonstrated that JQ1 treatment reduced the percentages of plasmablasts and plasma cells (data not shown).

To further determine the requirement of Brd4 for terminal B cell differentiation into plasma cells, we used an in vitrocostimulation method known to induce plasmablasts from peripheral B cells (anti-IgM $(5 \mu \mathrm{g} / \mathrm{ml})$, rCD40L $(1 \mu \mathrm{g} / \mathrm{ml})$, CpG ODN2006 $(5 \mu \mathrm{M})$, rIL-10 $(50 \mathrm{ng} / \mathrm{ml})$ and rIL-15 $(10 \mathrm{ng} / \mathrm{ml}))$ and examined the effect of PFI-1. We observed that during 5 days in culture, costimulation resulted in an increase in the percentage of $\mathrm{CD}_{1} 9^{+} \mathrm{IgD}^{-} \mathrm{CD} 27^{++}$and $\mathrm{CD} 19^{+} \mathrm{CD} 38^{++} \mathrm{CD} 138^{+}$, and this increase could be decreased by treatment with PFI-1 (Fig. 2C and D). Similar results were also obtained over 7 days in culture (Fig. S3). Collectively, our findings suggest that Brd4 inhibition modulates plasmablast-mediated plasma cell differentiation. Furthermore, we evaluated the effect of PFI-1 on the secretion of Ig. Analysis of culture supernatants revealed that costimulation increased the secretion of IgG and IgM; however, this increase was suppressed by treatment with PFI-1 (Fig. 2E).

Effect of Brd4 deficiency on efficient plasma cell differentiation in mice with B cell-specific deletion of the Brd4 gene

To further evaluate the in vivo function of Brd4 in modulating plasma cell differentiation, Brd4-CKO mice were generated by using the Cre-LoxP system (Fig. $3 \mathrm{~A}$ and B). Brd4 flox mice (Brd4 $4^{\mathrm{f} / \mathrm{f}}$, designated hereafter as "FL/FL" mice) were bred with CD19-Cre mice to generate B cell-specific deletion of Brd4 mice (Brd4 $4^{\mathrm{f} / \mathrm{f}}$, CD19 cre/-, hereafter designated as "CKO" mice). Mice carrying Brd4 $4^{\mathrm{f} / \mathrm{f}}$ and CD19-Cre (Brd4 4 f/f, CD19 ${ }^{\text {cre } /-}$ ) were born at the expected Mendelian ratio and developed normally when housed in a pathogen-free facility. RT-qPCR confirmed the tissue-specific deletion of Brd4 in B cells (Fig. 3C).

To determine the requirement of Brd4 for terminal B cell differentiation into plasma cells, the ability of Brd4 
deficient B cells to form plasma cells in vitro was assessed. B cells isolated from the spleen, lymph nodes and PBMCs were induced to differentiate using lipopolysaccharide (LPS, $10 \mu \mathrm{g} / \mathrm{mL}$ ) and IL-4 (10 ng/mL). We observed a reduced percentage of $\mathrm{CD} 138+$ plasma cells but not naïve $\mathrm{B}$ cells $\left(\mathrm{CD}_{19}{ }^{+} \mathrm{IgD}^{+} \mathrm{CD} 27^{-}\right)$or memory B cells $\left(\mathrm{CD}_{19}{ }^{+} \mathrm{IgD}^{-} \mathrm{CD} 27^{+}\right)$in Brd4-CKO mice compared with FL/FL (Fig. 3D, E, F, G). These findings provide more solid evidence for a critical role of Brd4 in regulating plasma cell differentiation.

\section{Brd4 modulate plasma cell differentiation through targeting BLIMP1}

BLIMP1 (encoded by prdm1) is a critical transcription factor that controls the terminal differentiation of B cells into plasma cells (Angelin-Duclos, Cattoretti et al., 2000; Radbruch et al., 2006). Therefore, to investigate how Brd4 controls differentiation of B cells into plasma cells, we firstly determined the effect of Brd4 inhibition on BLIMP1 expression in active CD19+ B cells. Purified CD19+ B cells were incubated with anti-IgM, rCD40L, CpG ODN2006, rIL-10 and rIL-15 for 7 days. We observed that treatment with PFI-1 or JQ1 reduced the costimulation-induced expression of BLIMP1 (Fig. 4A). We further evaluated the in vivo effect of Brd4 deficiency on the expression of BLIMPT1 in CD19+ B cells from Brd4-CKO mice. Isolated splenic B cells were stimulated with LPS $(10 \mu \mathrm{g} / \mathrm{mL})$ and IL-4 $(10 \mathrm{ng} / \mathrm{mL})$ for $48 \mathrm{~h}$. We found that the reduced expression of BLIMP1, but not prdm2, xbp-1 and CD69 in Brd4-CKO mice compared with WT mice (Fig. 4B). These data suggest that Brd4 controls the terminal differentiation of B cells into plasma cells by targeting BLIMP1.

Previous reports indicate that Brd4 can bind directly to transcription factors, therefore, to investigate how Brd4 modulates BLIMP1 expression, we determined whether Brd4 binds to and coordinately activates the BLIMP1 promoter. Because Brd4 has no regular binding site, we cloned the three BLIMP1 promoter regions (-2045-3207, -845-2087 and +333-883 bp from TSS) into a pGL4 - vectors, respectively, and measured luciferase activity in human 293 cells. As shown in Fig 4C, we demonstrated that transfection with Brd4 siRNA reduced the promoter activity of BLIMP1 in regions of -845-2087 bp (Blimp1-S2) and +333-883 bp (Blimp1-S3), suggesting that Brd4 is a critical factor in activating BLIMP1 promoter activity. To address whether Brd4 directly targets the BLIMP1 promoter, ChIP-qPCR assays were performed with primers located in the promoter region of BLIMP1 using anti-Brd4 antibody in B220 ${ }^{+} \mathrm{B}$ cells from spleen of FL/FL mice. We first used JASPAR DATABASE to predict both Brd4 binding site (-853 -838 and -487 -476) in BLIMP1 promoter (Fig. 4D). Then we demonstrated that Brd4 was significantly enriched in the BLIMP1 promoter region between -853 and -838 (Fig. 4E) in B220+ $\mathrm{B}$ cells from spleen of FL/FL mice, providing in vivo evidence that Brd4 directly binds and activates the endogenous BLIMP1 promoter.

Effect of Brd4 inhibition on the percentages of naïve, memory, plasmablast, and plasma cells in CD19+ B cells from patients with SLE

Based on our findings in healthy human B cell, we explored whether Brd4 inhibition regulates the differentiation of B cells from patients with SLE, a typical B cell-associated disorder. We first determined the percentages of naïve B cells, memory B cells and plasmablasts in healthy donors and patients with SLE. As shown in Fig.S4, SLE patients exhibited decreased percentages of naïve B cells, and increased percentages of memory B cells and plasmablast compared with healthy donors. We also found increased Brd4 expression in CD19+ B cell from SLE patients compared with those of HC subjects (Fig.S5). Next, we evaluated the effect of Brd4 inhibitors on the differentiation of B cells from SLE patients. Consistent with our findings from healthy donors, we also observed that treatment with PFI-1 decreased the percentages of plasmablasts and plasma cells, but did not influence the percentages of naïve and memory B cells (Fig.5A and B). We further examined the effect of PFI-1 treatment on plasmablast differentiation using an in vitro costimulation method known to induce plasmablasts from peripheral B cells. We observed that, during 7 days in culture, the costimulation resulted in an increase of the percentage of plasmablasts $\left(\mathrm{CD} 19^{+} \mathrm{IgD}^{-} \mathrm{CD} 38^{++}\right)$, and this increase could be decreased by treatment with PFI-1 (Fig.5C and D). In addition, we found that Brd4 inhibitors did not affect the proliferation and apoptosis of CD19+ B cells from patients with SLE (Fig.5E and $\mathrm{F})$.

The Brd4 inhibitor PFI-1 reduces hypergammaglobulinemia, plasma cells and attenuates ne- 


\section{phritis in MRL/lpr mice}

Hypergammaglobulinemia and the production of high-affinity IgG autoantibodies are important features of SLE, therefore, we first evaluated the effect of the Brd4 inhibitor PFI-1 on serum levels of IgG and its isotypes in MRL/lpr mice. As shown in Fig. 6A, PFI-1 significantly reduced total IgG, IgG1, and IgG2a levels already after 6 weeks of treatment. We further found that serum levels of anti-ANA and anti-dsDNA IgG were decreased in PFI-1-treated mice compared with vehicle-treated mice (Fig. S6). In contrast, serum levels of complement C3 and C4 were increased in PFI-1-treated mice (Fig. S6). PFI-1 treatment markedly decreased glomerular IgG and C3 deposits, and significantly ameliorated mesangial matrix expansion, mesangial cell proliferation and perivascular injuries as well as tubulointerstitial inflammatory cell infiltration and lesions (Fig. 6B and C). This improvement in renal histology was associated with a significant reduction in proteinuria (Fig. 6D) and serum creatinine and BUN levels (Fig. 6E). These findings suggest a critical role of Brd4 in controlling the production of autoantibodies and the progression of lupus nephritis.

To further evaluate the effect of PFI-1 on the frequency of B cell subsets in MRL/lpr mice, B cells were isolated from lymph nodes, PBMCs and spleens of mice. Flow cytometric analysis showed a significant reduction of plasma cells (Fig. 6F and G) but not memory B cells (Fig. S7) from lymph node, spleen and PBMCs of PFI-1-treated MRL/lpr mice compared with DMSO-treated mice. Moreover, as shown in Fig. $6 \mathrm{H}, \mathrm{PFI}-1$ treatment also reduced the expression of BLIMP1, a critical transcription factor that regulates plasma cell differentiation. These data provide in vivo evidence for the role of Brd4 in controlling plasma cell differentiation.

\section{Effect of Brd4-CKO on nephritis and percentages of plasma cells in mice with pristane-induced lupus model}

Pristane-induced glomerulonephritis in C57BL/6 mice is similar to the same autoantibodies typically observed in human lupus. We next evaluated the severity of nephritis in pristane-induced WT and Brd4-CKO mice. We observed that pristane-induced proteinuria was reduced in Brd4-CKO mice compared to WT mice (Fig. 6I). We further demonstrated an attenuation of kidney histologic damage using light microscopic examination in Brd4-CKO mice, as evidenced by a reduction in glomerular size, inhibition of mesangial expansion, and reduced cellularity (Fig. 6J).

To determine the impact of Brd4 deficiency on pristane-induced hypergammaglobulinemia, serum was collected at baseline and every month after pristine administration for 6 months. The serum levels of total $\operatorname{IgG}$, IgG1, and IgG2a were significantly reduced in Brd4-CKO mice compared to WT (FL/FL) mice at 6 months after pristane injection (Fig. S8).

To further evaluate the effect of Brd4 deficiency on the frequency of plasma cells in mice with pristaneinduced lupus model, B cells were isolated from lymph node, PBMCs and spleen of mice. Flow cytometric analysis showed significant reduction of plasma cells $\left(\mathrm{CD} 19^{+/-} \mathrm{CD} 138^{+}\right)$from the lymph nodes, PBMC and spleens of Brd4-CKO mice compared to WT (FL/FL) mice (Fig. 6K).

\section{DISCUSSION AND CONCLUSION}

In the present study, we show that Brd4 regulates plasma cell differentiation and immunoglobulin production by, at least in part, modulating BLIMP1 expression at the transcriptional level but does not affect the proliferation or apoptosis of activated primary CD19+ B cells from healthy controls or mice with B cell-specific deletion of Brd4. Brd4 inhibition also reduces the in vitro differentiation of plasma cells and the production of immunoglobulin in patients with SLE. The Brd4 inhibitor PFI-1 reduces hypergammaglobulinemia and the percentage of plasma cells and attenuates nephritis in MRL/lprmice. The inhibitory effect of Brd4 deficiency on nephritis and the percentages of plasmablasts and plasma cells is also shown in B cell-specific deletion of Brd4 mice with pristane-induced lupus. Collectively, our findings suggest that Brd4 plays a key role in regulating plasma cell differentiation and that Brd4 inhibitors have therapeutic potential for B cell-associated autoimmune disorders such as SLE.

Antibody production is dependent on the generation and maintenance of antibody-secreting cells, including 
plasmablasts and plasma cells, from their activated B cells. Short-lived plasmablasts are rapidly secreted effector cells of the early antibody response, whereas long-lived plasma cells are critical mediators of the lasting humoural immune response. However, the regulation of plasma cell differentiation is clearly complex and remains to be defined. Recent studies indicate that BET proteins are important in regulating Th17 cell differentiation (Cheung, Zhang et al., 2017; Mele et al., 2013). Brd4 has also been related to the IGH locus in B-cell lymphomas(Donato, Croci et al., 2017). These findings prompted us to speculate whether Brd4 is involved in B cell differentiation. In this work, we found that Brd4 suppression with a specific inhibitor or shRNA reduced the percentages of plasmablasts and plasma cells and the production of immunoglobulin in activated CD19+ B cells from healthy humans. By using mice with B cell-specific deletion of the Brd4 gene, we further observed that Brd4 deficiency reduced plasma cell differentiation and immunoglobulin secretion. These findings suggest a critical role of Brd4 in modulating plasma cell differentiation. Consistent with our results, a previous report showed that Brd4 plays an important role in regulating immunoglobulin gene expression from human CLNH11.4 B cells(Shim, Lee et al., 2017). Since the induction of apoptosis and the inhibition of proliferation in activated B cells may contribute to the reduction in plasma cell differentiation and immunoglobulin production, we determined the effect of Brd4 inhibition on the proliferation and apoptosis of B cells. However, we observed that Brd4 inhibition did not influence the proliferation and apoptosis of activated CD19+ B cells, suggesting that the effect of Brd4 inhibition on plasma cell differentiation is not associated with the proliferation and apoptosis of B cells. However, inconsistent with our findings, a recent report indicated that JQ1 affects the survival and proliferation of murine B cells(Kong, Rimes et al., 2020).

The differentiation of activated B cells into plasma cells is associated with alterations in gene expression that result in the loss of $\mathrm{B}$ cell identity and the gain of protein secretion functions. The master transcription factor BLIMP-1 plays a critical role in controlling B lymphocytes to a plasma cell fate(Angelin-Duclos et al., 2000; Nutt et al., 2011). In the B cell lineage, BLIMP1 is almost exclusively expressed in ASCs, with higher expression in long-lived plasma cells and lower expression in plasmablasts(Kallies, Hasbold et al., 2004). In the present study, we observed that costimulation with anti-IgM, rCD40L, CpG ODN2006, rIL-10 and rIL-15 for 7 days could upregulate BLIMP1 expression in CD19+ B cells from human heathy controls, and this increase was reduced by Brd4 inhibition. A similar result was obtained in CD19+ B cells from Brd4CKO mice compared with WT mice. These data suggest that Brd4 modulates plasma cell differentiation through, at least in part, regulating BLIMP1 expression. Furthermore, we investigated how Brd4 regulates BLIMP1 expression and found that Brd4 directly binds and activates the endogenous BLIMP1 promoter and thereby promotes its expression. These findings are consistent with previous studies showing that Brd4 binds directly and regulates other transcription factors, such as acetyl-310 RelA of nuclear factor kappa B (Sun, Wang et al., 2015) and c-Myc (Ba, Long et al., 2018). It is well known that Brd4 can interact with positive transcription elongation factor $\mathrm{b}(\mathrm{P}-\mathrm{TEFb})$ and recruit $\mathrm{P}-\mathrm{TEFb}$ to the promoter (Z. Yang, Yik et al., 2005; Zhang, Prakash et al., 2012). Elevated Brd4 expression induces an increase in the P-TEFb-dependent phosphorylation of RNA polymerase II CTD and the transcriptional activation of genes(Devaiah, Lewis et al., 2012). Therefore, based on our findings, we infer that Brd4 recruits P-TEFb to the BLIMP1 promoter, then results in the P-TEFb-dependent phosphorylation of RNA polymerase II, and finally promotes BLIMP1 expression. Collectively, our findings suggest that Brd4 controls plasma cell differentiation by, at least in part, directly regulating BLIMP1 transcription.

It has been established that B cells play a central role in both the onset and maintenance of autoimmunity in SLE. Recent studies indicate the significant clinical benefit of B cell depletion on the disease (Leandro, Edwards et al., 2002; Looney, Anolik et al., 2004; Reddy et al., 2015). An increased frequency of plasmablasts and plasma cells in peripheral blood has been demonstrated in active lupus patients (Arce, Jackson et al., 2001; Odendahl, Jacobi et al., 2000; C. Wei, Anolik et al., 2007). In the present study, we observed that Brd4 inhibition decreased the frequency of plasma cells and the production of immunoglobulin in costimulationstimulated CD19+ B cells from patients with SLE, indicating the role of Brd4 inhibition in regulating plasma cell differentiation in patients with SLE. Furthermore, to determine thein vivo effect of Brd4 inhibition on SLE, PFI-1 was used to administer the lupus animal model MRL/lpr mice. We found that PFI-1 treatment decreases hypergammaglobulinemia and the levels of autoantibodies and attenuates nephritis in MRL/lpr 
mice, which is consistent with a previous report that JQ1 treatment improves nephritis in MRL/lpr mice (S. Wei, Sun et al., 2015). More interestingly, the percentages of plasma cells and the expression of BLIMP1 were significantly reduced in B cells isolated from lymph nodes and spleens of PFI-1-treated MRL/lpr mice compared with the control group. We also demonstrate that nephritis is improved in B cell-specific deletion of Brd4 mice in a pristane-induced lupus model. Autoreactive plasma cells contribute to the maintenance of autoimmunity in SLE by the persistent secretion of autoantibodies (Hiepe, Dörner et al., 2011); therefore, our findings suggest that the suppression of Brd4 in plasma cell differentiation may contribute to its therapeutic efficacy in MRL/lpr mice. However, we do not rule out the possibility that Brd4 inhibition may have other molecular and cellular effects in vivo, since previous observations have shown the role of Brd4 inhibitors in modulating the differentiation and activation of Th17 and dendritic cells (Mele et al., 2013; Toniolo et al., 2015).

In conclusion, we identified Brd4 as an essential factor in regulating plasmablasts -mediated plasma cell differentiation and suggest that Brd4 is a target for the treatment of B cell-associated autoimmune disorders.

\section{ACKNOWLEDGMENTS}

This work was supported by grants from the National Natural Science Foundation of China (81701611, 81871275, U1401222), Guangzhou Science and Technology Project ( 201803010042), and Guangdong Basic and Applied basic Research Foundation (2020A1515010221).

\section{AUTHOR CONTRIBUTIONS}

Z.S., and Q.Q. performed the majority of the experiments and analyzed and interpreted the data. Z.Y., L.R., W.C., X.Y., L.M. and K.Y. collected clinical samples and analyzed and interpreted the data. X.S. and S.M. performed the animal experiments. Z.S., L.L., C. X and H.X. contributed to the study concept and design. Z.Y., L.L. and H.X. drafted the manuscript. C.X., L.L. and H.X. contributed to study supervision.

\section{CONFLICT OF INTEREST}

The authors have declared that no conflict of interest exists.

DECLARATION OF TRANSPARENCY AND SCIENTIFIC

RIGOUR

DECLARATION OF TRANSPARENCY AND SCIENTIFIC

\section{RIGOUR}

\section{DECLARATION OF TRANSPARENCY AND SCIENTIFICRIGOUR}

This Declaration acknowledges that this paper adhered to theprinciples for transparent reporting and scientific rigour of preclinical research as stated in the BJP guidelines for Design \& Analysis, Immunoblotting and Immunochemistry, and Animal Experimentation, and as recommended by funding agencies, publishers and otherorganizations engaged with supporting research

\section{DATA AVAILABILITY STATEMENT}

The authors declare that all supporting data are available in the article.

DECLARATION OF TRANSPARENCY AND SCIENTIFIC

\section{RIGOUR}

\section{REFERENCES}

Angelin-Duclos, C., Cattoretti, G., Lin, K. I., \& Calame, K. (2000). Commitment of B lymphocytes to a plasma cell fate is associated with Blimp-1 expression in vivo. J Immunol, 165 (10), 5462-5471. doi:10.4049/jimmunol.165.10.5462 
Arce, E., Jackson, D. G., Gill, M. A., Bennett, L. B., Banchereau, J., \& Pascual, V. (2001). Increased frequency of pre-germinal center B cells and plasma cell precursors in the blood of children with systemic lupus erythematosus. J Immunol, 167 (4), 2361-2369. doi:10.4049/jimmunol.167.4.2361

Ba, M., Long, H., Yan, Z., Wang, S., Wu, Y., Tu, Y., ... Cui, S. (2018). BRD4 promotes gastric cancer progression through the transcriptional and epigenetic regulation of c-MYC. J Cell Biochem, 119 (1), 973982. doi: $10.1002 /$ jcb. 26264

Bandukwala, H. S., Gagnon, J., Togher, S., Greenbaum, J. A., Lamperti, E. D., Parr, N. J., .. Rao, A. (2012). Selective inhibition of CD4+ T-cell cytokine production and autoimmunity by BET protein and c-Myc inhibitors. Proc Natl Acad Sci U S A, 109 (36), 14532-14537. doi:10.1073/pnas.1212264109

Belkina, A. C., Blanton, W. P., Nikolajczyk, B. S., \& Denis, G. V. (2014). The double bromodomain protein Brd2 promotes B cell expansion and mitogenesis. J Leukoc Biol, 95 (3), 451-460. doi:10.1189/jlb.1112588

Cappione, A., 3rd, Anolik, J. H., Pugh-Bernard, A., Barnard, J., Dutcher, P., Silverman, G., \& Sanz, I. (2005). Germinal center exclusion of autoreactive B cells is defective in human systemic lupus erythematosus. J Clin Invest, 115 (11), 3205-3216. doi:10.1172/jci24179

Carsetti, R., Rosado, M. M., \& Wardmann, H. (2004). Peripheral development of B cells in mouse and man. Immunol Rev, 197 , 179-191. doi:10.1111/j.0105-2896.2004.0109.x

Cheung, K. L., Zhang, F., Jaganathan, A., Sharma, R., Zhang, Q., Konuma, T., .. Zhou, M. M. (2017). Distinct Roles of Brd2 and Brd4 in Potentiating the Transcriptional Program for Th17 Cell Differentiation.Mol Cell, 65 (6), 1068-1080.e1065. doi:10.1016/j.molcel.2016.12.022

Devaiah, B. N., Lewis, B. A., Cherman, N., Hewitt, M. C., Albrecht, B. K., Robey, P. G., ... Singer, D. S. (2012). BRD4 is an atypical kinase that phosphorylates serine2 of the RNA polymerase II carboxy-terminal domain. Proc Natl Acad Sci U S A, 109 (18), 6927-6932. doi:10.1073/pnas.1120422109

Donato, E., Croci, O., Sabò, A., Muller, H., Morelli, M. J., Pelizzola, M., \& Campaner, S. (2017). Compensatory RNA polymerase 2 loading determines the efficacy and transcriptional selectivity of JQ1 in Myc-driven tumors. Leukemia, 31 (2), 479-490. doi:10.1038/leu.2016.182

Filippakopoulos, P., Picaud, S., Mangos, M., Keates, T., Lambert, J. P., Barsyte-Lovejoy, D., ... Knapp, S. (2012). Histone recognition and large-scale structural analysis of the human bromodomain family. Cell, 149 (1), 214-231. doi:10.1016/j.cell.2012.02.013

Grimaldi, C. M., Cleary, J., Dagtas, A. S., Moussai, D., \& Diamond, B. (2002). Estrogen alters thresholds for B cell apoptosis and activation.J Clin Invest, 109 (12), 1625-1633. doi:10.1172/jci14873

Grimaldi, C. M., Michael, D. J., \& Diamond, B. (2001). Cutting edge: expansion and activation of a population of autoreactive marginal zone B cells in a model of estrogen-induced lupus. J Immunol, 167 (4), 1886-1890. doi:10.4049/jimmunol.167.4.1886

Hiepe, F., Dörner, T., Hauser, A. E., Hoyer, B. F., Mei, H., \& Radbruch, A. (2011). Long-lived autoreactive plasma cells drive persistent autoimmune inflammation. Nat Rev Rheumatol, 7 (3), 170-178. doi:10.1038/nrrheum.2011.1

Hochberg, M. C. (1997). Updating the American College of Rheumatology revised criteria for the classification of systemic lupus erythematosus.Arthritis Rheum, 40 (9), 1725. doi:10.1002/art.1780400928

Kallies, A., Hasbold, J., Tarlinton, D. M., Dietrich, W., Corcoran, L. M., Hodgkin, P. D., \& Nutt, S. L. (2004). Plasma cell ontogeny defined by quantitative changes in blimp-1 expression. J Exp Med, 200 (8), 967-977. doi:10.1084/jem.20040973

Kong, I. Y., Rimes, J. S., Light, A., Todorovski, I., Jones, S., Morand, E., .. H Hawkins, E. D. (2020). Temporal Analysis of Brd4 Displacement in the Control of B Cell Survival, Proliferation, and Differentiation. Cell Rep, 33 (3), 108290. doi:10.1016/j.celrep.2020.108290 
Leandro, M. J., Edwards, J. C., Cambridge, G., Ehrenstein, M. R., \& Isenberg, D. A. (2002). An open study of B lymphocyte depletion in systemic lupus erythematosus. Arthritis Rheum, 46 (10), 2673-2677. doi:10.1002/art.10541

Looney, R. J., Anolik, J. H., Campbell, D., Felgar, R. E., Young, F., Arend, L. J., .. Sanz, I. (2004). B cell depletion as a novel treatment for systemic lupus erythematosus: a phase I/II dose-escalation trial of rituximab. Arthritis Rheum, 50 (8), 2580-2589. doi:10.1002/art.20430

Mele, D. A., Salmeron, A., Ghosh, S., Huang, H. R., Bryant, B. M., \& Lora, J. M. (2013). BET bromodomain inhibition suppresses TH17-mediated pathology. J Exp Med, 210 (11), 2181-2190. doi:10.1084/jem.20130376

Nutt, S. L., Hodgkin, P. D., Tarlinton, D. M., \& Corcoran, L. M. (2015). The generation of antibody-secreting plasma cells. Nat Rev Immunol, 15 (3), 160-171. doi:10.1038/nri3795

Nutt, S. L., Taubenheim, N., Hasbold, J., Corcoran, L. M., \& Hodgkin, P. D. (2011). The genetic network controlling plasma cell differentiation. Semin Immunol, 23 (5), 341-349. doi:10.1016/j.smim.2011.08.010

Odendahl, M., Jacobi, A., Hansen, A., Feist, E., Hiepe, F., Burmester, G. R., . . Dörner, T. (2000). Disturbed peripheral B lymphocyte homeostasis in systemic lupus erythematosus. J Immunol, 165 (10), 5970-5979. doi:10.4049/jimmunol.165.10.5970

Parodis, I., Stockfelt, M., \& Sjöwall, C. (2020). B Cell Therapy in Systemic Lupus Erythematosus: From Rationale to Clinical Practice.Front Med (Lausanne), 7 , 316. doi:10.3389/fmed.2020.00316

Radbruch, A., Muehlinghaus, G., Luger, E. O., Inamine, A., Smith, K. G., Dörner, T., \& Hiepe, F. (2006). Competence and competition: the challenge of becoming a long-lived plasma cell. Nat Rev Immunol, 6 (10), 741-750. doi:10.1038/nri1886

Reddy, V., Cambridge, G., Isenberg, D. A., Glennie, M. J., Cragg, M. S., \& Leandro, M. (2015). Internalization of rituximab and the efficiency of B Cell depletion in rheumatoid arthritis and systemic lupus erythematosus. Arthritis Rheumatol, 67 (8), 2046-2055. doi:10.1002/art.39167

Santulli-Marotto, S., Qian, Y., Ferguson, S., \& Clarke, S. H. (2001). Anti-Sm B cell differentiation in Ig transgenic MRL/Mp-lpr/lpr mice: altered differentiation and an accelerated response. J Immunol, 166 (8), 5292-5299. doi:10.4049/jimmunol.166.8.5292

Shapiro-Shelef, M., Lin, K. I., McHeyzer-Williams, L. J., Liao, J., McHeyzer-Williams, M. G., \& Calame, K. (2003). Blimp-1 is required for the formation of immunoglobulin secreting plasma cells and pre-plasma memory B cells. Immunity, 19 (4), 607-620. doi:10.1016/s1074-7613(03)00267-x

Shim, J. M., Lee, J. S., Russell, K. E., Wiegman, C. H., Barnes, P. J., Fear, D., ... Durham, A. L. (2017). BET proteins are a key component of immunoglobulin gene expression. Epigenomics, 9 (4), 393-406. doi:10.2217/epi-2016-0147

Shlomchik, M. J., \& Weisel, F. (2012). Germinal center selection and the development of memory B and plasma cells. Immunol Rev, 247 (1), 52-63. doi:10.1111/j.1600-065X.2012.01124.x

Sun, Y., Wang, Y., Toubai, T., Oravecz-Wilson, K., Liu, C., Mathewson, N., ... Reddy, P. (2015). BET bromodomain inhibition suppresses graft-versus-host disease after allogeneic bone marrow transplantation in mice. Blood, 125 (17), 2724-2728. doi:10.1182/blood-2014-08-598037

Toniolo, P. A., Liu, S., Yeh, J. E., Moraes-Vieira, P. M., Walker, S. R., Vafaizadeh, V., ... Frank, D. A. (2015). Inhibiting STAT5 by the BET bromodomain inhibitor JQ1 disrupts human dendritic cell maturation. J Immunol, 194 (7), 3180-3190. doi:10.4049/jimmunol.1401635

Wei, C., Anolik, J., Cappione, A., Zheng, B., Pugh-Bernard, A., Brooks, J., .. Sanz, I. (2007). A new population of cells lacking expression of CD27 represents a notable component of the B cell memory compartment in systemic lupus erythematosus. J Immunol, 178 (10), 6624-6633. doi:10.4049/jimmunol.178.10.6624 
Wei, S., Sun, Y., \& Sha, H. (2015). Therapeutic targeting of BET protein BRD4 delays murine lupus. Int Immunopharmacol, 29 (2), 314-319. doi:10.1016/j.intimp.2015.10.036

Yang, H., Wang, H., \& Jaenisch, R. (2014). Generating genetically modified mice using CRISPR/Casmediated genome engineering. Nat Protoc, 9 (8), 1956-1968. doi:10.1038/nprot.2014.134

Yang, Z., Yik, J. H., Chen, R., He, N., Jang, M. K., Ozato, K., \& Zhou, Q. (2005). Recruitment of P-TEFb for stimulation of transcriptional elongation by the bromodomain protein Brd4. Mol Cell, 19 (4), 535-545. doi:10.1016/j.molcel.2005.06.029

Yurasov, S., Tiller, T., Tsuiji, M., Velinzon, K., Pascual, V., Wardemann, H., \& Nussenzweig, M. C. (2006). Persistent expression of autoantibodies in SLE patients in remission. J Exp Med, 203 (10), 2255-2261. doi:10.1084/jem.20061446

Yurasov, S., Wardemann, H., Hammersen, J., Tsuiji, M., Meffre, E., Pascual, V., \& Nussenzweig, M. C. (2005). Defective B cell tolerance checkpoints in systemic lupus erythematosus. J Exp Med, 201 (5), 703711. doi:10.1084/jem.20042251

Zhang, W., Prakash, C., Sum, C., Gong, Y., Li, Y., Kwok, J. J., .. Chin, K. C. (2012). Bromodomaincontaining protein 4 (BRD4) regulates RNA polymerase II serine 2 phosphorylation in human CD4+ T cells.J Biol Chem, 287 (51), 43137-43155. doi:10.1074/jbc.M112.413047

\section{Figure legends}

Fig. 1 Effect of Brd4 inhibition on proliferation and apoptosis of activated human CD19+ B cells. (A ) Effect of Brd4 inhibitor on survival of activated B cells. Purified B cells were stimulated with combinations of antiIgM, rCD40L, CpG ODN2006, rIL-4 and rIL-2 as indicated in the presence of various concentration of Brd4 inhibitor PFI-1. At the time points indicated, the cells were stained with annexin V/PI. Percentages of viable cells (double negative for annexin $\mathrm{V}$ and PI) are shown as mean \pm SEM of five independent experiments. (B and C ) Effect of Brd4 inhibitor on proliferation of activated B cells. Purified CD19+ B cells labeled with CFSE were cultured with combinations of anti-IgM, rCD40L, CpG ODN2006, rIL-4 and rIL-2 in the presence of various concentration of PFI-1. After 7 days in culture, CD19+ B cells were analyzed for CFSE dilution. Panel B shown are representative of results. Data are mean \pm SEM of five independent experiments (C ). (D and E ) Effect of Brd4 inhibitor on apoptosis of activated B cells. Purified B cells were cultured with no stimulus (nil), co-stimulation (anti-IgM, rCD40L, CpG ODN2006, rIL-4 and rIL-2) in the presence of various concentrations of PFI-1. At the time points indicated, cells were washed and stained with annexin V and PI and analyzed by flow cytometry. The numbers in the lower right quadrants represent the percentage of apoptotic cells in culture (D ). Data are mean \pm SEM of of five independent experiments (E ). ${ }^{*} P<0.05$ versus nil (no co-stimulation)

Fig. 2 Effect of Brd4 inhibition on B cell differentiation and Ig production. (A and B ) Purified human CD19+ B cells were cultured with anti-IgM, rCD40L, CpG ODN2006, rIL-4 and rIL-2 as indicated in the presence of various concentration of Brd4 inhibitor PFI-1. The generation of naïve B cells $\left(\mathrm{CD} 19^{+} \mathrm{IgD}^{+} \mathrm{CD} 27^{-}\right)$, memory B cells $\left(\mathrm{CD} 19^{+} \mathrm{IgD}^{-} \mathrm{CD} 27^{+}\right)$, plasmablasts $\left(\mathrm{CD} 19^{+} \mathrm{IgD}^{-} \mathrm{CD} 27^{++}\right)$and plasma cells $\left(\mathrm{CD} 19^{+} \mathrm{CD} 38^{++} \mathrm{CD} 138^{+}\right)$ was assessed after 5 days in culture. The cells were stained for various surface markers and analyzed by flow cytometry. The numbers in the quadrants represent the percentage of cells in culture (A ). Data are mean \pm SEM of five independent experiments (B ). (C and D ) Effect of PFI-1 on differentiation of plasmablast and plasma cells. Purified human CD19+ B cells were cultured with anti-IgM $(5 \mu \mathrm{g} / \mathrm{ml}), \mathrm{rCD} 40 \mathrm{~L}(1 \mu \mathrm{g} / \mathrm{ml})$, CpG ODN2006 $(5 \mu \mathrm{M}), \mathrm{rIL}-10(50 \mathrm{ng} / \mathrm{ml})$ and $\mathrm{rIL}-15(10 \mathrm{ng} / \mathrm{ml})$. The percentage of plasmablast and plasma cells were examined after 5 days in culture by flow cytometry. Data are mean \pm SEM of five independent experiments (D ). (E ) IgG and IgM production was detected by ELISA. The results are the mean \pm SEM and are representative of five independent experiments. ${ }^{*} P<0.05$ versus nil (no co-stimulation), $\# P<0.05$ versus co-stim without PFI-1

Fig. 3 The inhibitory effect of Brd4 deficiency on plasma cell differentiation in mice with deletion of Brd4 in CD19+ B cells.(A ) Schematic representation of the endogenous Brd4 locus, targeting 
vector, locus following homologous recombination, FLP-mediated deletion of the neomycin resistance cassette, and Cre-mediated deletion of start codon-containing exon 5. (B ) Genotyping the allele with loxP sites using the DNA templates isolated from mice tails. The loxP site was amplified with the primers F3 and R3; the 300-bp band indicates the allele with loxP sites, and the 211-bp band indicates the WT allele. (C) The Brd4 CKO in CD19+ B cells was verified at the gene level with the splenic CD19+ B cells using RT-qPCR assay. ( $D-\boldsymbol{G})$ Effect of Brd4 deficiency on differentiation of naïve B cells $\left(\mathrm{CD} 19^{+} \mathrm{IgD}^{+} \mathrm{CD} 27^{-}\right.$, $\mathrm{D}$ and $\mathrm{E})$, memory B cells $\left(\mathrm{CD} 19^{+} \mathrm{IgD}^{-} \mathrm{CD} 27^{+}, \mathrm{D}\right.$ and $\left.\mathrm{E}\right)$ and $\mathrm{CD} 138+$ plasma cells $(\mathrm{F}$ and $\mathrm{G})$. Isolated splenic CD19+ B cells were induced to differentiate using lipopolysaccharide (LPS, $10 \mu \mathrm{g} / \mathrm{mL}$ ) and IL-4 (10 $\mathrm{ng} / \mathrm{mL}$ ) for $72 \mathrm{~h}$. The percentage of naïve B cells, memory B cells and plasma cells were examined by flow cytometry. Data are mean \pm SEM from 6 independent experiments (E and G ). The data are the mean \pm SEM and are representative of 6 independent experiments.

Fig. 4 Brd4 binds and activates the BLIMP1 promoter

(A ) Effect of Brd4 inhibition on the expression of BLIMP1 in human CD19+ B cells. Human CD19+ B cells were cultured with anti-IgM, rCD40L, CpG ODN2006, rIL-10 and rIL-15 in the presence or absence of PFI-1 or JQ1 for 7 days. BLIMP1 expression was measured by qRT-PCR. Data are expressed as mean \pm SEM from 5 independent experiments. (B ) Effect of Brd4 deficiency on expression of BLIMP1 in CD19+ B cells from Brd4-CKO mice. Isolated splenic B cells were stimulated with LPS (10 $\mu \mathrm{g} / \mathrm{mL})$ and IL-4 (10 $\mathrm{ng} / \mathrm{mL}$ ) for $48 \mathrm{~h}$. Data are expressed as mean \pm SEM of 6 independent experiments. (C) HEK-293T cells were cotransfected with BLIMP1 reporter and Brd4 siRNA or control siRNA. Luciferase activity was measured 48 hours post-transfection. (D) The potential Brd4 binding sites were evaluated by ChIP assay using anti-Brd4 or anti-IgG antibodies in $\mathrm{B} 220^{+} \mathrm{B}$ cells from spleen of wild type (FL/FL) mice. The presence of Brd4 binding site on BLIMP1 promotor was measured by qPCR. ${ }^{*} P<0.05,{ }^{* *} P<0.01$ versus control (ctrl) or wild type (FL/FL) or $\mathrm{siC},{ }^{\#} P<0.05$ versus co-stim.

Fig. 5 The effects of Brd4 inhibition on B cell differentiation and Ig production in SLE. (A and B ) Purified CD19+ B cells from SLE patients $(n=18)$ were cultured with anti-IgM, rCD40L, CpG ODN2006, rIL-4 and rIL-2 as indicated in the presence of Brd4 inhibitor PFI-1. The generation of naïve B cells $\left(\mathrm{CD} 19^{+} \mathrm{IgD}^{+} \mathrm{CD} 27^{-}\right)$, memory B cells $\left(\mathrm{CD} 19^{+} \mathrm{IgD}^{-} \mathrm{CD} 27^{+}\right)$, plasmablasts $\left(\mathrm{CD} 19^{+} \mathrm{IgD}^{-} \mathrm{CD} 27^{++}\right)$and plasma cells $\left(\mathrm{CD} 19^{+} \mathrm{CD} 38^{++} \mathrm{CD} 138^{+}\right)$was evaluated after 7 days in culture. The cells were stained for various surface markers and analyzed by flow cytometry. The numbers in the quadrants represent the percentage of cells in culture (A ). Data are mean \pm SEM (B ). (C and D) The effects of Brd4 inhibitor on differentiation of plasmablasts and plasma cells from SLE patients $(n=5)$. Purified CD19+ B cells were cultured with anti-IgM $(5 \mu \mathrm{g} / \mathrm{ml}), \mathrm{rCD} 40 \mathrm{~L}(1 \mu \mathrm{g} / \mathrm{ml})$, CpG ODN2006 $(5 \mu \mathrm{M}), \mathrm{rIL}-10(50 \mathrm{ng} / \mathrm{ml})$ and rIL-15 $(10 \mathrm{ng} / \mathrm{ml})$. The percentage of plasmablasts and plasma cells were examined after 5 days in culture by flow cytometry. Data are mean \pm SEM from five independent experiments (D ). (E and F ) Effect of Brd4 inhibition on apoptosis (E) and proliferation (F ) of activated lupus CD19+ B cells. Purified B cells were cultured with no stimulus (nil), co-stimulation (anti-IgM, rCD40L, CpG ODN2006, rIL-4 and rIL-2) in the presence of PFI-1 for 7 days. For detection of apoptosis, cells were washed and stained with annexin V and PI and analyzed by flow cytometry. For measurement of proliferation, cells were analyzed for CFSE dilution. (G ) IgG and IgM production detected by ELISA. The results are the mean \pm SEM from 8 independent experiments. ${ }^{*} P$ $<0.05$ vernus no stim, ${ }^{\#} P<0.05$ vernus co-stim without PFI-1

Fig 6 Effect of Brd4 inhibitor PFI-1 on hypergammaglobulinemia and nephritis in MRL/lpr mice MRL/lpr mice (12 weeks of age) were treated with vehicle (DMSO) or $2 \mathrm{mg} / \mathrm{kg}$ of PFI-1 for 6 weeks ( $\mathrm{n}=8$ mice per group). (A ) Effect of PFI-1 on s erum levels of total IgG, IgG1, IgG2a and IgM. Serum samples obtained from 6-month-old MRL/lpr mice were used as the standard control. (B and $\mathbf{C}$ ) Effect of PFI-1 on glomerulonephritis of MRL/lpr mice. Representative kidney sections stained with PAS (B) and frozen sections stained for IgG and C3 deposition $(\mathrm{C})$. Original magnification $\times 40$. (D) Urinary protein levels in mice at 12, 14, 16 and 18 weeks of age. (E) Serum creatinine (Cr) and blood urea nitrogen (BUN) levels at the end of the experiment. Bars in $\mathbf{A}, \mathbf{D}$ and $\mathbf{E}$ show the mean \pm SEM. ${ }^{*} P<0.05$ vs DMSO. (F and G ) Effect of Brd4 inhibitor PFI-1 on the percentages of plasma cells from spleen, lymph node and 
peripheral blood mononuclear cells (PBMC) of MRL/lpr mice. The cell surface markers of B cell subsets were analyzed by flow cytometry. The numbers are the percentages of cells within the quadrants. The bars represent the mean \pm SEM. ${ }^{*} P<0.05$ vs DMSO. (H ) Effect of PFI-1 on the BLIMP1 expression of splenic $\mathrm{B}$ cell from MRL/lpr mice. BLIMP1 expression was determined by quantitative RT-PCR. Data represent the mean \pm SEM from 8 mice. ${ }^{*} P<0.05,{ }^{* *} P<0.01$ vs DMSO. (I-K ) Effect of Brd4 deficiency on nephritis and percentages of plasma cells in Brd4-CKO mice with pristane-induced lupus model. (I) Urinary protein levels in FL/FL mice (FL/FL, $\mathrm{n}=6$ ), pristane-injected CKO mice (CKO-SLE, $\mathrm{n}=6)$, and pristane-injected FL/FL mice (FL/FL-SLE, $\mathrm{n}=6$ ) at 0,3 and 6 months after pristane injection. (J) Representative kidney sections from CKO and FL/FL mice stained with hematoxylin and eosin (H\&E) for analysis of glomerular size and mesangial expansion. (K ) Effect of Brd4 deficiency on frequency of plasma cell in mice with pristaneinduced lupus model. The frequency of plasma cells from spleen (SP), lymph node (LN) and peripheral blood mononuclear cells (PBMC) was evaluated using flow cytometric analysis. The numbers are the percentages of cells within the quadrants. The bars represent the mean \pm SEM. ${ }^{*} P<0.05$ versus FL/FL-SLE.

\section{Hosted file}

image-1.pdf available at https://authorea.com/users/407798/articles/518018-the-suppressionof-brd4-inhibits-peripheral-plasma-cell-differentiation-and-exhibits-therapeuticpotential-for-systemic-lupus-erythematosus 